\title{
international wound journal 9
}

\section{Severe Postoperative Wound Healing Disturbance in a Patient with Alpha-1-Antitrypsin deficiency: The Impact of Augmentation Therapy}

\begin{tabular}{|c|c|}
\hline Journal: & International Wound Journal \\
\hline Manuscript ID: & IWJ-14-322.R1 \\
\hline Wiley - Manuscript type: & Original Article \\
\hline Keywords: & Alpha-1-antitrypsin deficiency, Wound healing disturbance, Panniculitis \\
\hline Abstract: & $\begin{array}{l}\text { Background: Wound healing disturbance is a common complication } \\
\text { following surgery, but the underlying cause sometimes remains elusive. } \\
\text { Case presentation: A } 50 \text { years old Caucasian male developed an initially } \\
\text { misunderstood severe wound healing disturbance following colon and } \\
\text { abdominal wall surgery. An untreated alpha-1-antitrypsin deficiency in the } \\
\text { patient's medical history, known since } 20 \text { years and clinically apparent as a } \\
\text { mild to moderate chronic obstructive pulmonary disease, was eventually } \\
\text { found to be at its origin. Further clinical work-up showed alpha-1- } \\
\text { antitrypsin serum levels below } 30 \% \text { of the lower reference value, } \\
\text { phenotype testing revealed a ZZ phenotype and a biopsy taken from the } \\
\text { wound area showed the characteristic, disease-related histological pattern } \\
\text { of necrotising panniculitits. Augmentation therapy with plasma alpha-1- } \\
\text { antitrypsin was initiated and within a few weeks, rapid and adequate would } \\
\text { healing was observed. } \\
\text { Conclusion: Alpha-1-antitrypsin deficiency is an uncommon but clinically } \\
\text { significant, possible cause of wound healing disturbances. An augmentation } \\
\text { therapy ought to be considered in affected patients during the } \\
\text { perioperative period. }\end{array}$ \\
\hline
\end{tabular}




\section{Background}

Wound healing disturbance is a frequent postoperative complication, generally caused by a combination of surgery-related factors and patient's condition including duration of the intervention, contamination of the operative field or wound site, age, nutritional status and other risk factors such as regular intake of steroids or immunosuppressants as well as smoking and cardiovascular disease entailing tissue hypoxia. At the other end of the spectrum, Alpha-1-antitrypsin (AAT) deficiency has been described as a rare cause of impaired wound healing ${ }^{1}$.

AAT deficiency is the most commonly inherited metabolic disease, mainly predisposing to liver and lung injury. AAT is an abundantly circulating serine protease inhibitor that basically neutralizes neutrophil elastase ${ }^{2}$. In case of reduction or absence of AAT the inhibition of elastase and subsequent prevention of tissue destruction is diminished. According to the mobility of the protein inhibitor in the electrophoresis, four different phenotypes can be differentiated referred to as M (medium), S (slow), Z (very slow) and null phenotype ${ }^{3}$. The MM phenotype represents the wildtype, whereas the $\mathrm{ZZ}$ is associated with the severest clinical progression ${ }^{4,5}$. The $\mathrm{ZZ}$ variant results in normal synthesis of AAT, but only $15 \%$ of the produced enzyme are secreted from the hepatocytes ${ }^{6}$. Clinical symptoms can be observed when the serum concentration of AAT is below $35 \%$ of the average standard level ${ }^{7}$. According to the phenotype, the prevalence of this autosomal recessive disorder varies between 0.015 and $0.04 \%$, accounting for an estimated number of up to $296^{\prime} 000$ affected patients in the entire Europe $\mathrm{e}^{4,8-10}$. The most common clinical manifestations of AAT deficiency are panacinar pulmonary emphysema and liver cirrhosis ${ }^{11,12}$. However, AAT deficiency associated necrotising panniculitis has been described in the literature as a rare, but specific cause of delayed wound healing ${ }^{13,14}$.

We present a case of a patient suffering from a severe postoperative wound healing disturbance due to an AAT deficiency associated panniculitis. The pertinent literature will be reviewed and discussed. 


\section{Case report}

A 53 year old Caucasian male patient was urgently hospitalised at Bern University Hospital and a steroid treatment (Methylprednisolon $125 \mathrm{mg} / \mathrm{d}$ ) for an assumed systemic capillary leak syndrome (Clarkson's disease) was initiated. Patient's history revealed a poor nutritional status and a chronic obstructive pulmonary disease (Gold II, $\mathrm{FEV}_{1}=57 \%$ of the standard). The latter possibly being attributed to the AAT deficiency diagnosed 20 years ago, yet untreated since then. After three days of therapy, the patient developed an acute abdomen requiring emergency laparotomy. A perforation of the caecum with fecal peritonitis was encountered, and an ileo-caecal resection with an abdominal split (ileum/ascending colon) ostomy were performed via an open laparotomy.

Owing to an ankylosing spondylitis, the patient was receiving regular (every eight weeks) infusions of the immunosuppressant infliximab, the last dose dating back 48 hours before admission. At time of first surgery, a spontaneous blister of unknown origin was located at the right side of the abdomen. After its debridement, an open, fibrin-coated wound remained.

Eight days after primary surgery the patient developed a complete dehiscence of the laparotomy. Abdominal wall closure was achieved using an intra-peritoneal onlay mesh (IPOM), and a vacuumassisted device (VAC) was installed at a continuous negative pressure of $25 \mathrm{mmHg}$ in order to enhance wound healing. The VAC was subsequently changed three times a week. However, neither the laparotomy wound nor the debrided blister in the flank showed any sign of granulation tissue (Figure $1 \mathrm{a}$ and $\mathrm{b}, 1 \mathrm{f}$ and $\mathrm{g})$. Even worse, further skin lesions developed, one in the right lower abdomen beneath the ostomy, another upon the sacrum, both reminding the clinical aspects of the blister and the laparotomy wounds. The wounds in the right flank and upon the sacrum were each dressed using a VAC device. 
Given the utter absence of wound healing for more than ten weeks, extended diagnostic evaluation for connective tissue disease, vasculitis and anti-phospholipid syndrome as well as serologic testing for various viral infections including human immuno-deficiency virus (HIV) and hepatitis were performed. None of these analyses yielded further clues about the aetiology of the pending wound healing disorder. A biopsy taken from the blister wound in the right flank revealed a necrotising panniculitis.

AAT serum concentration was found to be significantly decreased to $0.25 \mathrm{~g} / \mathrm{L}$ (standard values: 0.90 to $2.00 \mathrm{~g} / \mathrm{L}$ ), and genetic testing showed a $\mathrm{ZZ}$ phenotype. Based on these findings, augmentation therapy using AAT (Prolastin®, Crucell, Bern, Switzerland, 60mg per kilogram weekly) was started. Within 7 days after initiation of the treatment, significant granulation tissue and wound healing could be observed (Figure 1c). After eight weeks of periodic ATT infusions, serum concentration was within standard values at $0.92 \mathrm{~g} / \mathrm{L}$, and all VAC devices could eventually be removed definitely (Figure $1 \mathrm{~d}$ and e). Another ten weeks later the skin lesions were all completely cured and the patient was discharged to a rehabilitation centre.

\section{Conclusion}

AAT deficiency is typically characterised by pulmonal emphysema and liver cirrhosis ${ }^{15}$. Necrotising panniculitis was shown to be a possible complication of AAT deficiency. AAT associated panniculitis typically presents with recurrent painful, red nodules or plaques, suppurated ulcerations and expression of oily discharge from the skin lesions refractory to usual treatment, namely to steroids ${ }^{7,16}$. Occurrence of the inflammation is often related to antecedent trauma or excessive activity, and predilection sites are thighs, buttock and trunk ${ }^{3}$. Histology is characterised through abundant neutrophil granulocytes and lobular fat necrosis ${ }^{17}$. Histological overhaul of the wound biopsy from the 
blister's ground in the right flank showed the typical signs of necrotising panniculitis. In the present case, two skin lesions can be ascribed to traumatic conditions, the laparotomy to the surgical intervention and the sacral wound to the bedriddenness. The blister in the right flank, however, could not be attributed to a specific traumatic event.

Differential diagnosis includes wound healing disturbance due to high-dose steroid or infliximab therapy and malnutrition, respectively. However, steroid therapy was discontinued after three days of application and the wound repair failure persisted more than 10 weeks after weaning of the steroid therapy. Infliximab is reported to lose its effect within 4 to 8 weeks after administration ${ }^{18}$. This casts steroids and infliximab as a possible causes for the wound healing disturbance into doubt. Furthermore, short-term application ( $<10$ days) of even high doses of steroids do not seem be a risk factor for impaired wound healing ${ }^{19}$. Initial nutrient deficiency was substituted by parenteral nutrition and enteral feeding via a naso-jejunal tube (at admission: Albumin 23g/L; reference values: 35 $52 \mathrm{~g} / \mathrm{L}$ ). Serum albumin were found to be within standard levels 39 days after the initial operation.

The $\mathrm{ZZ}$ phenotype determined by the genetic studies in this patient is generally associated with the severest clinical manifestation of AAT deficiency, including lung and liver disease as well as panniculitis and wound healing disturbances ${ }^{20}$. While a liver affection was never diagnosed in our patient, he is suffering from several diseases that could be associated with AAT deficiency, including chronic obstructive pulmonary disease (Gold stadium II), hypothyroidism, ankolysing spondylitis, and panniculitis ${ }^{13}$. However, the pathogenesis of panniculitis in the context of AAT deficiency remains unknown and there is no correlation described between serologic AAT values and the occurrence of panniculitis. In the literature even some patients with phenotype MS, who have normal AAT serum levels, were reported to develop panniculits ${ }^{16}$. 
In the presented case, intravenous substitution of human purified enzyme (Prolastin®) entailed formation of granulation tissue at one week, and normalisation of serum levels of AAT, at three weeks after starting the therapy, respectively. The persistent wound healing failure whilst AAT deficiency was untreated and prompt formation of granulation tissue after onset of the augmentation therapy suggests respective causality. Appropriate histology as well as serum concentration of AAT of below $30 \%$ of the lower reference value prior to treatment further support the diagnosis of AAT deficiency related wound healing impairment. Successful augmentation therapy of AAT deficiency associated panniculits has been described earlier ${ }^{21}$. While other cases reported in the literature were treated with either tetracycline, steroids, non-steroidal anti-inflammatory drugs or hydroxychloroquine, individualized Prolastin ${ }^{\circledR}$ therapy in terms of starting time of application and dosage showed to be the most effective treatment strategy ${ }^{22}$. To date, no adverse effects of AAT substitution are documented, but no long-term data are available ${ }^{23}$.

In summary, AAT deficiency should be considered as a possible cause of wound healing impairment, particularly if lack of the enzyme is already recorded in patient's medical history. Furthermore, when intractable perioperative wound healing disturbance of unknown origin persists AAT deficiency should be considered as differential diagnosis. This case report represents, to our knowledge, the first published case of AAT deficiency related wound healing problems after surgery. Diagnosis is made based on AAT serum levels, genetic test and the occurrence of histologically proven panniculitis. The pathogenesis of fat tissue inflammation in the context of AAT deficiency has not yet been elucidated. Yet, intravenous Prolastin ${ }^{\circledR}$ substitution seems to be the most effective treatment strategy. We therefore suggest to assess AAT serum levels in patients with a known deficiency prior to surgical intervention. If criteria of AAT deficiency are met, preventive substitution therapy should be considered to avoid postoperative wound healing failure. 
Figure 1: Laparostomy and wound area of the blister after debridement. No granulation tissue has occurred until initiating augmentation therapy. Pictures show the chronology of the wound healing, including number of days after the first surgery (1. digit) and the time before and after the beginning of the substitution (2. digit, in days). a) $+32 /-41$, b) $+55 /-18$, c $)+86 /+13$, d) $+107 /+34$, e) $+142 /+69$, f) $+28 /-45$, g) $+55 /-18$, h) $+79 /+6$, i) $+107 /+34, \mathrm{k})+142 /+69$.

\section{Acknowledgments}

The authors declare that they have no competing interests. 


\section{References}

1. Guo S, Dipietro LA. Factors affecting wound healing. Journal of dental research 2010;89:21929.

2. Sun Z, Yang P. Role of imbalance between neutrophil elastase and alpha 1-antitrypsin in cancer development and progression. The lancet oncology 2004;5:182-90.

3. Geraminejad P, DeBloom JR, 2nd, Walling HW, Sontheimer RD, VanBeek M. Alpha-1antitrypsin associated panniculitis: the MS variant. Journal of the American Academy of Dermatology 2004;51:645-55.

4. Kohnlein T, Welte T. Alpha-1 antitrypsin deficiency: pathogenesis, clinical presentation, diagnosis, and treatment. The American journal of medicine 2008;121:3-9.

5. Ioachimescu OC, Stoller JK. A review of alpha-1 antitrypsin deficiency. Copd 2005;2:263-75.

6. Alpha 1-antitrypsin deficiency: memorandum from a WHO meeting. Bulletin of the World Health Organization 1997;75:397-415.

7. Valverde R, Rosales B, Ortiz-de Frutos FJ, Rodriguez-Peralto JL, Ortiz-Romero PL. Alpha-1antitrypsin deficiency panniculitis. Dermatologic clinics 2008;26:447-51, vi.

8. Dawwas MF, Davies SE, Griffiths WJ, Lomas DA, Alexander GJ. Prevalence and risk factors for liver involvement in individuals with PiZZ-related lung disease. American journal of respiratory and critical care medicine 2013;187:502-8.

9. Tzakis A. Early recognition of alpha-1 antitrypsin deficiency and considerations for liver transplantation. Gastroenterology \& hepatology 2013;9:110-2.

10. de Serres FJ, Blanco I. Prevalence of alpha1-antitrypsin deficiency alleles $\mathrm{PI} * \mathrm{~S}$ and $\mathrm{PI} * \mathrm{Z}$ worldwide and effective screening for each of the five phenotypic classes $\mathrm{PI} I^{*} \mathrm{MS}, \mathrm{PI} I^{*} \mathrm{MZ}, \mathrm{PI} \mathrm{SS}^{*} \mathrm{PI} \mathrm{SZ}^{*}$, and PI*ZZ: a comprehensive review. Therapeutic advances in respiratory disease 2012;6:277-95.

11. Kelly E, Greene CM, Carroll TP, McElvaney NG, O'Neill SJ. Alpha-1 antitrypsin deficiency. Respiratory medicine 2010;104:763-72.

12. Fregonese L, Stolk J. Hereditary alpha-1-antitrypsin deficiency and its clinical consequences. Orphanet journal of rare diseases 2008;3:16.

13. Stone H, Pye A, Stockley RA. Disease associations in alpha-1-antitrypsin deficiency. Respiratory medicine 2013.

14. Wood AM, Stockley RA. Alpha one antitrypsin deficiency: from gene to treatment. Respiration; international review of thoracic diseases 2007;74:481-92.

15. Silverman EK, Sandhaus RA. Clinical practice. Alpha1-antitrypsin deficiency. The New England journal of medicine 2009;360:2749-57.

16. Ortiz PG, Skov BG, Benfeldt E. Alpha1-antitrypsin deficiency-associated panniculitis: case report and review of treatment options. Journal of the European Academy of Dermatology and Venereology : JEADV 2005;19:487-90.

17. Su WP, Smith KC, Pittelkow MR, Winkelmann RK. Alpha 1-antitrypsin deficiency panniculitis: a histopathologic and immunopathologic study of four cases. The American Journal of dermatopathology 1987;9:483-90.

18. Goh L, Samanta A. Update on biologic therapies in ankylosing spondylitis: a literature review. Int J Rheum Dis 2012;15:445-54.

19. Wang AS, Armstrong EJ, Armstrong AW. Corticosteroids and wound healing: clinical considerations in the perioperative period. American journal of surgery 2013;206:410-7.

20. Stockley RA, Turner AM. alpha-1-Antitrypsin deficiency: clinical variability, assessment, and treatment. Trends in molecular medicine 2014;20:105-15.

21. Chowdhury MM, Williams EJ, Morris JS, et al. Severe panniculitis caused by homozygous ZZ alpha1-antitrypsin deficiency treated successfully with human purified enzyme (Prolastin). The British journal of dermatology 2002;147:1258-61.

22. Stockley RA, Miravitlles M, Vogelmeier C. Augmentation therapy for alpha-1 antitrypsin deficiency: towards a personalised approach. Orphanet journal of rare diseases 2013;8:149. 
Cathomas et al. p.8

23. Mohanka M, Khemasuwan D, Stoller JK. A review of augmentation therapy for alpha-1 antitrypsin deficiency. Expert opinion on biological therapy 2012;12:685-700. 


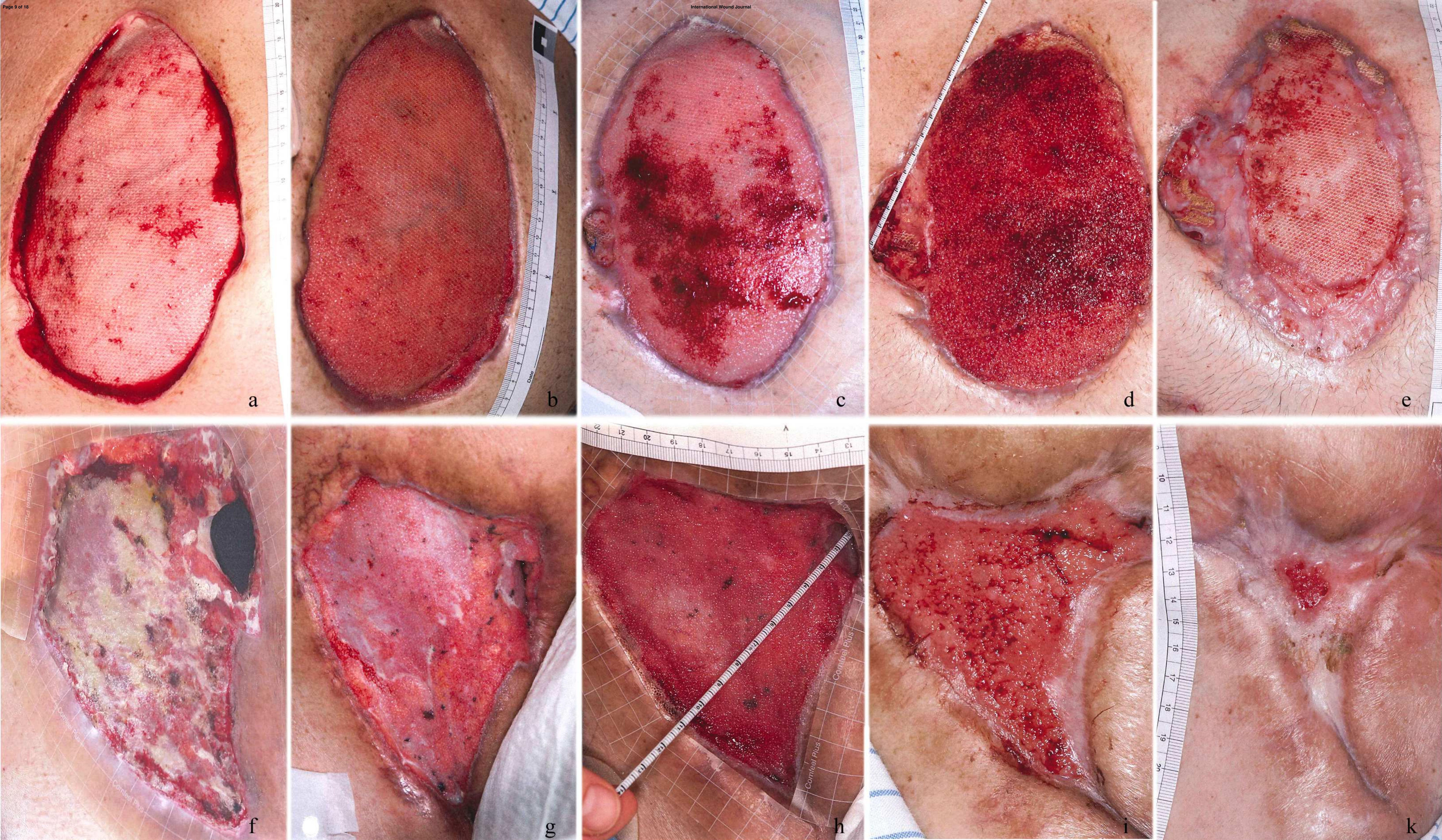




\section{Key Points}

Wound healing disturbance is a common complication following surgery. Sometimes the underlying reason remains elusive and no accurate therapy can be induced. In this report, a case of necrotising panniculitis due to an untreated alpha-1-antitrypsin deficiency is described. Within a few weeks after initiation of the augmentation therapy with plasma alpha-1-antitrypsin, an adequate wound healing was observed. This case should show a further cause for a wound healing disturbance, which can be easily treated. 


\section{Background}

Wound healing disturbance is a frequent postoperative complication, generally caused by a combination of surgery-related factors and patient's condition. These include duration of the intervention, contamination of the operative field or wound site, age, nutritional status and other risk factors such as regular intake of steroids or immunosuppressants as well as smoking and cardiovascular disease entailing tissue hypoxia. At the other end of the spectrum, Alpha-1-antitrypsin (AAT) deficiency has been described as a rare cause of impaired wound healing ${ }^{1}$.

AAT deficiency is the most commonly inherited metabolic disease, characteristically predisposing to liver and lung injury. AAT is an abundantly circulating serine protease inhibitor that neutralizes neutrophil elastase ${ }^{2}$. In case of reduction or absence of AAT the inhibition of elastase and subsequent prevention of tissue destruction is diminished. According to the mobility of the protein inhibitor in the electrophoresis, four different phenotypes can be differentiated referred to as M (medium), S (slow), Z (very slow) and null phenotype ${ }^{3}$. The MM phenotype represents the wildtype, whereas the $\mathrm{ZZ}$ is associated with the severest clinical progression ${ }^{4,5}$. The $\mathrm{ZZ}$ variant results in normal synthesis of AAT, but only $15 \%$ of the produced enzyme are secreted from the hepatocytes ${ }^{6}$. Clinical symptoms can be observed when the serum concentration of AAT is below $35 \%$ of the average standard level ${ }^{7}$. According to the phenotype, the prevalence of this autosomal recessive disorder varies between 0.015 and $0.04 \%$, accounting for an estimated number of up to $296^{\prime} 000$ affected patients in Europe $e^{4,8-10}$. The most common clinical manifestations of AAT deficiency are panacinar pulmonary emphysema and liver cirrhosis ${ }^{11,12}$. In addition, AAT deficiency associated necrotising panniculitis has been described in the literature as a rare, but specific cause of delayed wound healing ${ }^{13,14}$.

We present a case of a patient suffering from a severe postoperative wound healing disturbance due to an AAT deficiency associated panniculitis. The literature will be reviewed and discussed. 


\section{Case report}

A 53 year old Caucasian male patient was hospitalised at Bern University Hospital for acute renal failure, and steroid treatment (Methylprednisolon $125 \mathrm{mg} / \mathrm{d}$ ) for an assumed systemic capillary leak syndrome (Clarkson's disease) was initiated. Patient's history revealed a poor nutritional status and a chronic obstructive pulmonary disease (Gold II, $\mathrm{FEV}_{1}=57 \%$ of the standard), the latter possibly being attributed to an AAT deficiency diagnosed 20 years ago, yet untreated since then. After three days of therapy, the patient developed an acute abdomen requiring emergency laparotomy. A perforation of the caecum with fecal peritonitis was encountered, and an ileo-caecal resection with an abdominal split (ileum/ascending colon) ostomy were performed via an open laparotomy.

Owing to an ankylosing spondylitis, the patient was receiving regular (every eight weeks) infusions of the immunosuppressant infliximab, the last dose dating back 48 hours before admission. At time of first surgery, a spontaneous blister of unknown origin developed at the right side of the abdomen. After its debridement, an open, fibrin-coated wound remained.

Eight days after primary surgery, the patient developed a burst abdomen. Abdominal wall closure was achieved using an intra-peritoneal onlay mesh (IPOM), and a vacuum-assisted device (VAC) was installed at a continuous negative pressure of $25 \mathrm{mmHg}$ in order to enhance wound healing. The VAC was subsequently changed three times a week. However, neither the laparotomy wound nor the debrided blister in the flank showed any sign of granulation tissue (Figure 1a and b, 1f and g). Even worse, further skin lesions developed, one in the right lower abdomen beneath the ostomy, another upon the sacrum, both reminding the clinical aspects of the blister and the laparotomy wounds. The wounds in the right flank and upon the sacrum were each treated using a VAC device.

Given the utter absence of wound healing for more than ten weeks, extended diagnostic evaluation for connective tissue disease, vasculitis and anti-phospholipid syndrome as well as serologic testing for 
various viral infections including human immuno-deficiency virus (HIV) and hepatitis were performed. None of these analyses yielded further clues about the aetiology of the lack of wound healing disorder. A biopsy taken from the blister wound in the right flank revealed a necrotising panniculitis.

Finally, AAT serum concentration was tested and found to be significantly decreased to $0.25 \mathrm{~g} / \mathrm{L}$ (standard values: 0.90 to $2.00 \mathrm{~g} / \mathrm{L}$ ), and genetic testing confirmed a $\mathrm{ZZ}$ phenotype. Based on these findings, augmentation therapy using AAT (Prolastin ${ }^{\circledR}$, Crucell, Bern, Switzerland, 60mg per kilogram weekly) was started. Within 7 days after initiation of the treatment, significant granulation tissue developed and wound healing could be observed (Figure 1c). After eight weeks of periodic ATT infusions, serum concentration was within standard values at $0.92 \mathrm{~g} / \mathrm{L}$, and all VAC devices could be removed definitely (Figure $1 \mathrm{~d}$ and e). Another ten weeks later, the skin lesions were all completely cured, and the patient was discharged to a rehabilitation centre.

\section{Conclusion}

AAT deficiency is typically characterised by pulmonal emphysema and liver cirrhosis ${ }^{15}$. Necrotising panniculitis was shown to be a possible complication of AAT deficiency. AAT associated panniculitis typically presents with recurrent painful, red nodules or plaques, suppurated ulcerations and expression of oily discharge from the skin lesions refractory to usual treatment, namely to steroids ${ }^{7,16}$. Development of lesions is often related to antecedent trauma or excessive activity, and predilection sites are thighs, buttock and trunk ${ }^{3}$. Histology is characterised through abundant neutrophil granulocytes and lobular fat necrosis ${ }^{17}$. The histology of the wound biopsy from the blister's ground in the right flank showed the typical signs of necrotising panniculitis. In the present case, two skin lesions can be ascribed to traumatic conditions, the laparotomy to the surgical intervention and the 
sacral wound to the bedriddenness. The blister in the right flank, however, could not be attributed to a specific traumatic event.

Differential diagnosis includes wound healing disturbance due to high-dose steroid or infliximab therapy and malnutrition, respectively. Steroid therapy was applied for only three days, and the most recent dose of infliximab was administered 48 hours before the initial hospitalization. Furthermore, short-term application ( $<10$ days) of even high doses of steroids do not seem to be a risk factor for impaired wound healing ${ }^{19}$, and infliximab is reported to lose its effect within 4 to 8 weeks after administration ${ }^{18}$. The wound repair failure, however, persisted more than 10 weeks after weaning of the steroid and infliximab therapy, making the two drugs unlikely to be the cause of the persistent wound healing disturbance. Initial nutrient deficiency was substituted by parenteral nutrition and enteral feeding via a naso-jejunal tube (at admission: Albumin 23g/L; reference values: $35-52 \mathrm{~g} / \mathrm{L}$ ). Serum albumin was found to be within standard levels 6 weeks after the initial operation.

The $\mathrm{ZZ}$ phenotype determined by the genetic studies in this patient is generally associated with the severest clinical manifestation of AAT deficiency, including lung and liver disease as well as panniculitis and wound healing disturbances ${ }^{20}$. While a liver affection was never diagnosed in our patient, he is suffering from several diseases that could be associated with AAT deficiency, including chronic obstructive pulmonary disease (Gold stadium II), hypothyroidism, ankolysing spondylitis, and panniculitis ${ }^{13}$. However, the pathogenesis of panniculitis in the context of AAT deficiency remains unknown and there is no correlation described between serologic AAT values and the occurrence of panniculitis. In the literature even some patients with phenotype MS, who have normal AAT serum levels, were reported to develop panniculits ${ }^{16}$.

In the presented case, intravenous substitution of human purified enzyme (Prolastin $囚$ ) leads to the formation of granulation tissue after one week and normalisation of serum levels of AAT three weeks 
after starting therapy, respectively. The persistent wound healing failure whilst AAT deficiency was untreated and prompt formation of granulation tissue after onset of the augmentation therapy suggests respective causality. Appropriate histology as well as serum concentration of AAT of below $30 \%$ of the lower reference value prior to treatment further supports the diagnosis of AAT deficiency related wound healing impairment. Successful augmentation therapy in spontaneous occurring AAT deficiency associated panniculits lesions has been described ${ }^{21}$. While other cases reported in the literature were treated with either tetracycline, steroids, non-steroidal anti-inflammatory drugs or hydroxychloroquine, individualized Prolastin ${ }^{\circledR}$ therapy in terms of starting time of application and dosage showed to be the most effective treatment strategy ${ }^{22}$. To date, no adverse effects of AAT substitution are documented, but no long-term data are available ${ }^{23}$.

In summary, AAT deficiency should be considered as a possible cause of wound healing impairment, particularly if lack of the enzyme is already recorded in patient's medical history. Furthermore, when intractable perioperative wound healing disturbance of unknown origin persists, AAT deficiency should be considered in the differential diagnosis. This case report represents, to our knowledge, the first published case of AAT deficiency related wound healing problems after surgery. Diagnosis is made based on AAT serum levels, genetic test and the occurrence of histologically proven panniculitis. The pathogenesis of fat tissue inflammation in the context of AAT deficiency has not yet been elucidated. Yet, intravenous Prolastin ${ }^{\circledR}$ substitution seems to be the most effective treatment strategy. We therefore suggest to assess AAT serum levels in patients with a known deficiency prior to surgical intervention. If criteria of AAT deficiency are met, preventive substitution therapy should be considered to avoid postoperative wound healing failure.

Figure 1: Laparostomy and wound area of the blister after debridement. No granulation tissue has occurred until initiating augmentation therapy. Pictures show the chronology of the wound healing, 
including number of days after the first surgery (1. digit) and the time before and after the beginning of the substitution (2. digit, in days). a) $+32 /-41$, b $)+55 /-18$, c $)+86 /+13$, d) $+107 /+34$, e) $+142 /+69$, f) $+28 /-45$, g) $+55 /-18$, h) $+79 /+6$, i) $+107 /+34, \mathrm{k})+142 /+69$.

\section{Acknowledgments}

The authors declare that they have no competing interests. 


\section{References}

1. Guo S, Dipietro LA. Factors affecting wound healing. Journal of dental research 2010;89:21929.

2. Sun Z, Yang P. Role of imbalance between neutrophil elastase and alpha 1-antitrypsin in cancer development and progression. The lancet oncology 2004;5:182-90.

3. Geraminejad P, DeBloom JR, 2nd, Walling HW, Sontheimer RD, VanBeek M. Alpha-1antitrypsin associated panniculitis: the MS variant. Journal of the American Academy of Dermatology 2004;51:645-55.

4. Kohnlein T, Welte T. Alpha-1 antitrypsin deficiency: pathogenesis, clinical presentation, diagnosis, and treatment. The American journal of medicine 2008;121:3-9.

5. Ioachimescu OC, Stoller JK. A review of alpha-1 antitrypsin deficiency. Copd 2005;2:263-75.

6. Alpha 1-antitrypsin deficiency: memorandum from a WHO meeting. Bulletin of the World Health Organization 1997;75:397-415.

7. Valverde R, Rosales B, Ortiz-de Frutos FJ, Rodriguez-Peralto JL, Ortiz-Romero PL. Alpha-1antitrypsin deficiency panniculitis. Dermatologic clinics 2008;26:447-51, vi.

8. Dawwas MF, Davies SE, Griffiths WJ, Lomas DA, Alexander GJ. Prevalence and risk factors for liver involvement in individuals with PiZZ-related lung disease. American journal of respiratory and critical care medicine 2013;187:502-8.

9. Tzakis A. Early recognition of alpha-1 antitrypsin deficiency and considerations for liver transplantation. Gastroenterology \& hepatology 2013;9:110-2.

10. de Serres FJ, Blanco I. Prevalence of alpha1-antitrypsin deficiency alleles $\mathrm{PI} * \mathrm{~S}$ and $\mathrm{PI} * \mathrm{Z}$ worldwide and effective screening for each of the five phenotypic classes $\mathrm{PI} I^{*} \mathrm{MS}, \mathrm{PI} I^{*} \mathrm{MZ}, \mathrm{PI} \mathrm{SS}^{*} \mathrm{PI} \mathrm{SZ}^{*}$, and PI*ZZ: a comprehensive review. Therapeutic advances in respiratory disease 2012;6:277-95.

11. Kelly E, Greene CM, Carroll TP, McElvaney NG, O'Neill SJ. Alpha-1 antitrypsin deficiency. Respiratory medicine 2010;104:763-72.

12. Fregonese L, Stolk J. Hereditary alpha-1-antitrypsin deficiency and its clinical consequences. Orphanet journal of rare diseases 2008;3:16.

13. Stone H, Pye A, Stockley RA. Disease associations in alpha-1-antitrypsin deficiency. Respiratory medicine 2013.

14. Wood AM, Stockley RA. Alpha one antitrypsin deficiency: from gene to treatment. Respiration; international review of thoracic diseases 2007;74:481-92.

15. Silverman EK, Sandhaus RA. Clinical practice. Alpha1-antitrypsin deficiency. The New England journal of medicine 2009;360:2749-57.

16. Ortiz PG, Skov BG, Benfeldt E. Alpha1-antitrypsin deficiency-associated panniculitis: case report and review of treatment options. Journal of the European Academy of Dermatology and Venereology : JEADV 2005;19:487-90.

17. Su WP, Smith KC, Pittelkow MR, Winkelmann RK. Alpha 1-antitrypsin deficiency panniculitis: a histopathologic and immunopathologic study of four cases. The American Journal of dermatopathology 1987;9:483-90.

18. Goh L, Samanta A. Update on biologic therapies in ankylosing spondylitis: a literature review. Int J Rheum Dis 2012;15:445-54.

19. Wang AS, Armstrong EJ, Armstrong AW. Corticosteroids and wound healing: clinical considerations in the perioperative period. American journal of surgery 2013;206:410-7.

20. Stockley RA, Turner AM. alpha-1-Antitrypsin deficiency: clinical variability, assessment, and treatment. Trends in molecular medicine 2014;20:105-15.

21. Chowdhury MM, Williams EJ, Morris JS, et al. Severe panniculitis caused by homozygous ZZ alpha1-antitrypsin deficiency treated successfully with human purified enzyme (Prolastin). The British journal of dermatology 2002;147:1258-61.

22. Stockley RA, Miravitlles M, Vogelmeier C. Augmentation therapy for alpha-1 antitrypsin deficiency: towards a personalised approach. Orphanet journal of rare diseases 2013;8:149. 
Cathomas et al. p.8

23. Mohanka M, Khemasuwan D, Stoller JK. A review of augmentation therapy for alpha-1 antitrypsin deficiency. Expert opinion on biological therapy 2012;12:685-700. 